Publ. RIMS, Kyoto Univ.

12 (1976), 123-140

\title{
On the Dual of Besov Spaces
}

\author{
By
}

Tosinobu MURAMATU*)

\section{$\S$ 1. Introduction and Main Results}

This paper is a supplement to the author's paper [6]. Here we shall discuss the space $B_{p, \infty-}^{\sigma}(\Omega)$, a closed subspace of $B_{p, \infty}^{\sigma}(\Omega)$, and determine the dual of Besov spaces $B_{p, q}^{\sigma}(\Omega)$.

For a measure space $(M, \mu)$ and a Banach space $X$ by $L^{p}(M, \mu ; X)$ we denote the space of all $X$-valued strongly measurable functions $f(x)$ such that $\|f(x)\|_{X} \in L^{p}(M, \mu)$. For the sake of simplicity, we write $d_{*} y=|y|^{-m} d y$, where $y \in M \subset \boldsymbol{R}^{m}, L_{*}{ }^{p}(M ; X)=L^{p}\left(M, d_{*} y ; X\right), 1 \leqq p$ $\leqq \infty$, and by $L_{*}{ }^{\infty-}(M ; X)$ we denote the closed subspace of all functions $f \in L_{*}{ }^{\infty}(M ; X)$ which converge to zero as $|y| \rightarrow 0$ and as $|y| \rightarrow \infty$. We shall make use of the following conventions: $p<\infty-<\infty$ for real $p, 1 / \infty-=1 / \infty=0$.

The space $B_{p, \infty_{-}}^{\sigma}(\Omega ; X)$ is defined as follows:

Definition 1. 1. Let $\Omega$ be an open set in $\boldsymbol{R}^{n}$. For $0<\sigma<1$ $B_{p, \infty-}^{\sigma}(\Omega ; X)$ is the space of all functions $f \in L^{p}(\Omega ; X)$ such that

$$
\|f(x+y)-f(x)\|_{L^{p}\left(\Omega_{1, y} ; x\right)}|y|^{-\sigma} \in L_{*}{ }^{\infty-}\left(\boldsymbol{R}^{n}\right),
$$

where $\Omega_{j, y}=\{x ; x, x+y, \cdots, x+j y \in \Omega\}$, and $B_{p, \infty-}^{1}(\Omega ; X)$ is the space of all $f \in L^{p}(\Omega ; X)$ such that

$$
\|f(x+2 y)-2 f(x+y)+f(x)\|_{L^{p}\left(\Omega_{2, y} ; x\right)}|y|^{-1} \in L_{*}^{\infty-}\left(\boldsymbol{R}^{n}\right) .
$$

For $\sigma=k+\theta, 0<\theta \leqq 1, k$ is a positive integer, $B_{p, \infty-}^{\sigma}(\Omega ; X)$ is the space of all $f \in W_{p}{ }^{k}(\Omega ; X)$ whose all partial derivaties $D^{\alpha} f$ of order $k$ belong, to $B_{p, \infty-}^{\theta}(\Omega ; X)$. Finally, for $\sigma=k+\theta, 0<\theta \leqq 1, k$ is a negative integer,

Received October 15, 1975.

* Present address, Department of Mathematics, Tokyo University of Education, Tokyo 
$B_{p, \infty-}^{\sigma}(\Omega ; X)$ is the space of all $f$ which can be expressed as

$$
f=\sum_{|\alpha| \leqq-k} D^{\alpha} f_{\alpha}, f_{\alpha} \in B_{p, \infty-}^{\theta}(\Omega ; X)^{1)} .
$$

The space $B_{\infty, \infty-}^{1}\left(\boldsymbol{T}^{1}\right)$, where $\boldsymbol{T}^{1}$ is the 1-dimensional torus, is identical with the space of smooth functions due to Zygmund [7]. In his paper it is shown that the space $B_{p, \infty}^{1}$ plays an essential rôle in problems of the theory of real functions and of trigonometric series. Our notation $L_{*}{ }^{\infty-}$ is due to Komatsu [3].

As in [6] we assume throughout this paper that $\Omega$ is an open set with the cone property, and by $\Psi(x), t_{0}, b, \mathcal{K}_{j}$ we denote the same things as in [6] (see p. 328, p. 329).

Now, we state our main results. $B y B_{p, q, \bar{Q}}^{\sigma}\left(\boldsymbol{R}^{n}\right)$ we shall denote the closed subspace of all $f \in B_{p, q}^{\sigma}\left(\boldsymbol{R}^{n}\right)$ whose support is contained in $\bar{\Omega}$.

Theorem 1.2. If $1 \leqq p \leqq \infty, 1 \leqq q \leqq \infty, 1 / p+1 / p^{\prime}=1,1 / q+1 / q^{\prime}$ $=1$, and if $\sigma$ is a real number, then there exists unique continuous bilinear form $\langle f, g\rangle$ on $B_{p, q}^{\sigma}(\Omega) \times B_{p^{\prime}, q^{\prime}, \bar{Q}}^{-\sigma}\left(\boldsymbol{R}^{n}\right)$ with the following properties: (i) If $j>-\sigma, K \in \mathcal{K}_{j}, 0<a<t_{0}, u(t, x) \in L_{*}{ }^{q}\left([0, a] ; L^{p}(\Omega)\right)$, $K^{0} \in \mathcal{K}_{0}, f^{0}(x) \in L^{p}(\Omega)$, and if $g \in B_{p^{\prime}, q^{\prime}, \bar{\theta}}^{-\sigma}\left(\boldsymbol{R}^{n}\right)$, then

$$
\begin{aligned}
& \left\langle\int_{0}^{a} d_{*} t \int t^{\sigma-n} K\left(t, x, \frac{x-y}{t}, y\right) u(t, y) d y, g(x)\right\rangle \\
& \quad=\int_{0}^{a} d_{*} t \int t^{\sigma-n} u(t, y)\left\langle K\left(t, x, \frac{x-y}{t}, y\right), g(x)\right\rangle_{x} d y, \\
& \left\langle\int a^{-n} K^{0}\left(a, x, \frac{x-y}{a}, y\right) f^{0}(y) d y, g(x)\right\rangle \\
& =\int f^{0}(y)\left\langle a^{-n} K^{0}\left(a, x, \frac{x-y}{a}, y\right), g(x)\right\rangle_{x} d y .
\end{aligned}
$$

(ii) If $i>\sigma, H \in \mathcal{K}_{i}, v(t, x) \in L_{*}{ }^{q^{\prime}}\left([0, a] ; L^{p^{\prime}}(\Omega)\right), H^{0} \in \mathcal{K}_{0}, g^{0}(x)$ $\in L^{p^{\prime}}(\Omega)$, and if $f \in B_{p, q}^{\sigma}(\Omega)$, then

$$
\begin{aligned}
& \left\langle f(x), \int_{0}^{a} d_{*} t \int t^{-\sigma-n} H\left(t, y, \frac{y-x}{t}, x\right) v(t, y) d y\right\rangle \\
& =\int_{0}^{a} d_{*} t t^{-\sigma-n} v(t, y)\left\langle H\left(t, y, \frac{y-x}{t}, x\right), f(x)\right\rangle_{x} d y,
\end{aligned}
$$

1) $\alpha=\left(\alpha_{1}, \cdots, \alpha_{n}\right),|\alpha|=\alpha_{1}+\cdots+\alpha_{n}, D_{j}=\partial / \partial x_{j}, D^{\alpha}=D_{1}^{\alpha_{1}} \cdots D_{n}^{\alpha_{n}}$. 


$$
\begin{aligned}
& \left\langle f(x), \int a^{-n} H^{0}\left(a, y, \frac{y-x}{a}, x\right) g^{0}(y) d y\right\rangle \\
& \quad=\int g^{0}(y)\left\langle a^{-n} H^{0}\left(a, y, \frac{y-x}{a}, x\right), f(x)\right\rangle_{x} d y .
\end{aligned}
$$

Here $\langle\varphi(x), f(x)\rangle_{x}$ denotes the duality on $\mathscr{D}(\Omega) \times \mathscr{D}^{\prime}(\Omega)$.

Theorem 1.3. Let $\sigma, p, q, p^{\prime}, q^{\prime}$, and $\langle$,$\rangle be as in Theorem$ 1.2. Then $B_{p, q}^{\sigma}(\Omega)$ and $B_{p^{\prime}, q^{\prime}, \bar{\Omega}}^{-\sigma}\left(\boldsymbol{R}^{n}\right)$ form a dual pair with respect to $\langle$,$\rangle : (i) If g \in B_{p^{\prime}, q^{\prime}, \bar{\Omega}}^{-\sigma}\left(\boldsymbol{R}^{n}\right)$, and if $\langle f, g\rangle=0$ for all $f \in B_{p, q}^{\sigma}(\Omega)$, then $g=0$. (ii) If $f \in B_{p, q}^{\sigma}(\Omega)$, and if $\langle f, g\rangle=0$ for all $g \in B_{p^{\prime}, q^{\prime}, \bar{\Omega}}^{-\sigma}\left(\boldsymbol{R}^{n}\right)$, then $f=0$.

By Theorem 1.2 and Theorem 1.3 we observe that the mapping $g$ $\rightarrow l_{g}$, where $l_{g}(f)=\langle f, g\rangle$ for all $f \in B_{p, q}^{\sigma}(\Omega)$, is a continuous injection from $B_{p^{\prime}, q^{\prime}, \bar{\Omega}}^{-\sigma}\left(\boldsymbol{R}^{n}\right)$ into $\left\{B_{p, q}(\Omega)\right\}^{\prime}$ (the dual space of $B_{p, q}^{\sigma}(\Omega)$ ), and the mapping $f \rightarrow l_{f}$, where $l_{f}(g)=\langle f, g\rangle$ for all $g \in B_{p^{\prime}, q^{\prime}, \bar{\Omega}}^{\sigma}\left(\boldsymbol{R}^{n}\right)$, is a continuous injection from $B_{p, q}^{\sigma}(\Omega)$ into $\left\{B_{p^{\prime}, q^{\prime}, \bar{\Omega}}^{-\sigma}\left(\boldsymbol{R}^{n}\right)\right\}^{\prime}$. In [6] we proved that these mappings are surjective ([6] Theorem 9) if $1<p<\infty, 1<q$ $<\infty-$. But there are some other cases for which they are surjective. Namely,

Theorem 1.4. Let $p, q, p^{\prime}, q^{\prime}$, and $\sigma$ be as in Theorem 1.2. (i) The dual of $B_{p, q}^{\sigma}(\Omega)$ is canonically isomorphic to $B_{p^{\prime}, q^{\prime}, \bar{a}}^{\sigma}\left(\boldsymbol{R}^{n}\right)$, that is, the mapping $g \rightarrow l_{g}$ is an isomorphism if (a) $1 \leqq p<\infty, 1 \leqq q \leqq \infty-$, $q^{\prime} \neq \infty-$, or (b) $\Omega$ is bounded and $p=\infty, 1 \leqq q \leqq \infty-, q^{\prime} \neq \infty-$. The dual of $B_{p^{\prime}, q^{\prime}, \bar{Q}}^{-\sigma}\left(\boldsymbol{R}^{n}\right)$ is canonically isomorphic to $B_{p, q}^{\sigma}(\Omega)$, that is, the mapping $f \rightarrow l_{f}$ is an isomorphism if (a) $1 \leqq p^{\prime}<\infty, 1 \leqq q^{\prime} \leqq \infty-$, $q \neq \infty-$, or (b) $\Omega$ is bounded and $p^{\prime}=\infty, 1 \leqq q^{\prime} \leqq \infty-, p=1, q \neq \infty-$.

Some special cases of Theorem 1.4 are proved by Flett: The proof of the fact that $\left\{B_{p, 1}^{\sigma}\left(\boldsymbol{R}^{n}\right)\right\}^{\prime}=B_{p^{\prime}, \infty}^{-\sigma}\left(\boldsymbol{R}^{n}\right)$ for $1 \leqq p<\infty$, and $\left\{B_{p, \infty-}^{\sigma}\left(\boldsymbol{R}^{n}\right)\right\}^{\prime}$ $=B_{p, 1}^{-\sigma}\left(\boldsymbol{R}^{n}\right)$ for $1<p<\infty$, is given in [1], and that of $\left\{B_{p, 1}^{\sigma}\left(\boldsymbol{T}^{1}\right)\right\}^{\prime}$ $=B_{p^{\prime, \infty}}^{-\sigma}\left(\boldsymbol{T}^{1}\right)$ and $\left\{B_{p, \infty-}^{\sigma}\left(\boldsymbol{T}^{1}\right)\right\}^{\prime}=B_{p^{\prime}, 1}^{-\sigma}\left(\boldsymbol{T}^{1}\right)$ for $1 \leqq p \leqq \infty$, where $\boldsymbol{T}^{1}$ is the one-dimensional torus (circle), is given in [2]. 


\section{$\S$ 2. The Space $L^{q}\left(M, \mu ; L^{p}\right)$}

We shall begin with observing some properties of the space $L^{q}\left(M, \mu ; L^{p}\right)$ which is closely related to Besov spaces.

Lemma 2. 1. Let $(M, \mu)$ be a $\sigma$-finite measure space, $1 \leqq p \leqq \infty$, $\left\{E_{k}\right\}_{k=1,2, \ldots}$, be an increasing sequence of measurable sets of finite measure whose union is $M, f$ be a $\mu$-measurable function, and let $f_{k}(x)=\min$ $\{|f(x)|, k\} \chi_{k}(x)$, where $\chi_{k}$ is the characteristic function of $E_{k}$. Then $\left\|f_{k}\right\|_{L^{p}(M)} \rightarrow\|f\|_{L^{p}(M)}$ as $k \rightarrow \infty$.

Proof. It is obvious that $f_{k}(x) \rightarrow|f(x)|$ as $k \rightarrow \infty$ for every $x$. If $1 \leqq p<\infty$, then the assertion follows from Fatou's lemma. Assume now $p=\infty$, and let $l_{k}=\operatorname{ess} \sup f_{k}(x)$. Since $\left\{f_{k}(x)\right\} ; k=1,2, \cdots$, is increasing it follows that $l=\lim l_{k}$ exists. The fact that $f_{k}(x) \leqq|f(x)|$ implies that $l \leqq\|f\|_{L^{\infty}(M)}$. On the other hand, it is seen that $\left\{x ;|f(x)|=\lim f_{k}(x)\right.$ $>r\}=\cup_{k}\left\{x ; f_{k}(x)>r\right\}$, which gives the converse inequality. The proof is complete.

Lemma. 2. 2. Let $M$ be a measurable set in $\boldsymbol{R}^{n}, 1 \leqq_{p} \leqq \infty$, and let $1 / p+1 / p^{\prime}=1, p^{\prime} \neq \infty-$. If $f$ is a measurable non-negative function such that

$$
\int_{M} f(x) g(x) d_{*} x \leqq c\|g\|_{L_{*}^{p}(M)}
$$

holds for all non-negative function $g$ in $L_{*}{ }^{p}(M)$, then $f \in L_{*}{ }^{p^{\prime}}(M)$ and $\|f\|_{L_{*}^{p^{\prime}}} \leqq c$.

Proof. Let $E_{k}=M \cap\{x ; 1 / k<|x|<k\}, \chi_{k}$ be the characteristic function of $E_{k}$, and let $f_{k}(x)=\min \{f(x), k\} \chi_{k}(x)$. By Lemma 2.1 it is sufficient to prove that $\left\|f_{k}\right\|_{L_{*} p^{\prime}} \leqq c$.

(i) Case $p=1$. Let $\chi_{k r}$ be the characteristic function of the set $\{x$; $\left.f_{k}(x)>r\right\}$. Substituting $\chi_{k r}$ for $g$, by $(2 \cdot 1)$ we have

$$
\int r \chi_{k r}(x) d_{*} x \leqq \int \chi_{k r}(x) f(x) d_{*} x \leqq c \int \chi_{k r}(x) d_{*} x .
$$

Hence, for $r>c$ the measure of the set $\left\{x ; f_{k}(x)>r\right\}$ is equal to zero, 
that is, ess. $\sup f_{k}(x) \leqq c$.

(ii) Case $1<p<\infty-$ Taking $g(x)=f_{k}(x)^{p^{\prime-1}}$ we obtain

$$
\left(\left\|f_{k}\right\|_{L_{*} p^{\prime}}\right)^{p^{\prime}} \leqq \int f_{k}(x)^{p^{\prime}-1} f(x) d_{*} x \leqq c\left(\left\|f_{k}\right\|_{L_{*} p^{\prime}}\right)^{p^{\prime}-1},
$$

and hence $\left\|f_{k}\right\|_{L_{*}{ }^{p}} \leqq c$.

(iii) $p \geqq \infty-$. Since $\chi_{k} \in L_{*}{ }^{\infty-}$, taking $g=\chi_{k}$, we have by $(2 \cdot 1)$ that

$$
\left\|f_{k}\right\|_{L_{*^{1}}} \leqq \int \chi_{k}(x) f(x) d_{*} x \leqq c\left\|\chi_{k}\right\|_{L_{*}^{\infty}}=c .
$$

Lemma 2. 3. Let $\left(M_{1}, \mu\right)$ be $\sigma$-finite measure space, $M_{2}$ be a measurable set in $\boldsymbol{R}^{n}, 1 \leqq p, q \leqq \infty, 1 / p+1 / p^{\prime}=1,1 / q+1 / q^{\prime}=1, q^{\prime}$ $\neq \infty$, and let $g(x, y)$ be a measurable function. Then

$$
\left|\iint_{M_{1} \times M_{2}} f(x, y) g(x, y) \mu(d x) d_{*} y\right| \leqq C\|f\|_{L_{*}^{q}\left(M_{2} ; L^{p}\left(M_{1}\right)\right)}
$$

holds for all $f \in L_{*}{ }^{q}\left(M_{2} ; L^{p}\left(M_{1}\right)\right)$ if and only if

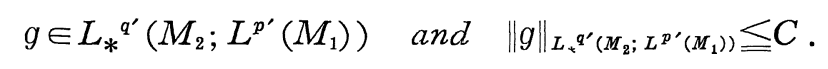

Proof. The fact that $(2 \cdot 3)$ implies $(2 \cdot 2)$ is a consequence of Hölder's inequality. Conversely assume that $(2 \cdot 2)$ holds for all $f$ in $L_{*}{ }^{q}\left(M_{2} ; L^{p}\left(M_{1}\right)\right)$. Let $\psi(y)=\|g(x, y)\|_{L^{p^{\prime}\left(M_{1}\right)}}$ if $g(x, y) \in L^{p^{\prime}}\left(M_{1}\right)$, and $\phi(y)=\infty$ otherwise. Then, it is sufficient to show that

$$
\psi \in L_{*}{ }^{q^{\prime}}\left(M_{2}\right) \text { and }\|\psi\|_{L_{\tau}{ }^{q^{\prime}}} \leqq C .
$$

To show this let $\left\{E_{k}\right\}$ be an incresing sequence of measurable set in $M_{1}$ such that $0<\mu_{1}\left(E_{k}\right)<\infty$ and $\cup E_{k}=M_{1}, g_{k}(x, y)=\min \{|g(x, y)|, k\}$ $\chi_{k}(x)$, where $\chi_{k}$ is the characteristic function of $E_{k}$, and let $\phi_{k}=\| g_{k}$ $(x, y) \|_{L^{p}\left(M_{1}\right)}$. Then, by Lemma $2.2(2 \cdot 4)$ follows from the fact that

$$
\int \varphi(y) \psi_{k}(y) d_{*} y \leqq C\|\varphi\|_{L_{*}{ }^{q}\left(M_{2}\right)}
$$

holds for any non-negative functions $\varphi$ in $L_{*}{ }^{q}\left(M_{2}\right)$. First consider the case where $p=1$. Let $0<\eta<1, E_{k, \eta}=\left\{(x, y) ; x \in E_{k}, g_{k}(x, y) \geqq \eta \psi_{k}(y)\right\}$, $\sigma_{k}(y)=\mu\left\{x ;(x, y) \in E_{k, \eta}\right\}$, and let $\chi_{k, \eta}$ be the characteristic function of $E_{k, \eta}$. For a complex number $\zeta$ let $e(\zeta)=|\zeta| / \zeta$ if $\zeta \neq 0$ and $e(0)=0$. Let $f(x, y)=\varphi(y) \sigma_{k}(y)^{-1} \chi_{k, \eta}(x, y) e(g(x, y))$. Then 


$$
\begin{aligned}
\eta \int \varphi(y) \psi_{k}(y) d_{*} y & \leqq \iint f(x, y) g(x, y) \mu(d x) d_{*} y \\
& \leqq C\|f\|_{L_{*}{ }^{q}\left(M_{2} ; L^{1}\left(M_{1}\right)\right)}=C\|\varphi\|_{L^{q}{ }^{q}\left(M_{2}\right)} .
\end{aligned}
$$

Therefore, letting $\eta \rightarrow 1$, we obtain (2.5). Next assume that $1<p<\infty$. Let $f(x, y)=\varphi(y) e(g(x, y))\left\{g_{k}(x, y) / \psi_{k}(y)\right\}^{p^{\prime}-1}$ if $\psi_{k}(y) \neq 0$, and $f(x, y)$ $=0$ if $\psi_{k}(y)=0$. Then we have

$$
\begin{aligned}
\int \varphi(y) \psi_{k}(y) d_{*} y & \leqq \iint f(x, y) g(x, y) \mu(d x) d_{*} y \\
& \leqq C\|f\|_{L_{*}{ }^{q}\left(M_{2}: L^{p}\left(M_{1}\right)\right)}=C\|\varphi\|_{L_{*}{ }^{q}\left(M_{2}\right)} .
\end{aligned}
$$

Finally let $f(x, y)=\chi_{k}(x) \varphi(y) e(g(x, y))$. Then we obtain (2.5) for the case $p=\infty$.

Lemma 2. 4. Let $\left(M_{1}, \mu_{1}\right),\left(M_{2}, \mu_{2}\right)$ be $\sigma$-finite measure spaces, $1 \leqq p, q \leqq \infty$, and let $X$ be a Banach space. Assume that $K(x, \xi, y, \eta)$ is a measurable function such that

$$
\begin{aligned}
& \sup _{y} \int|K(x, \xi, y, \eta)| \mu_{2}(d \eta), \sup _{\eta} \int|K(x, \xi, y, \eta)| \mu_{2}(d y) \leqq k(x, \xi), \\
& \sup _{x} \int k(x, \xi) \mu_{1}(d \xi), \sup _{\xi} \int k(x, \xi) \mu_{1}(d x) \leqq C<\infty .
\end{aligned}
$$

Then the operator defined by

$$
(T u)(x, y)=\iint K(x, \xi, y, \eta) u(\xi, \eta) \mu_{1}(d \xi) \mu_{2}(d \eta)
$$

is a bounded linear operator on $L^{q}\left(M_{1}, \mu_{1} ; L^{p}\left(M_{2}, \mu_{2} ; X\right)\right)$ with norm not greater than $C$.

Proof. This follows from Lemma 2.5 in [5].

Corollary 2.5. Let $\left(M_{1}, \mu_{1}\right),\left(M_{2}, \mu_{2}\right), p, q$ and $K$ be as in Lemma 2.4, and let $1 / p+1 / p^{\prime}=1 / q+1 / q^{\prime}=1$. If $u \in L^{q}\left(M_{1}, \mu_{1} ; L^{p}\left(M_{1}, \mu_{2}\right)\right)$ and $v \in L^{q^{\prime}}\left(M_{1}, \mu_{1} ; L^{p^{\prime}}\left(M_{2}, \mu_{2}\right)\right)$, then the integral

$$
\iiint \int K(x, \xi, y, \eta) u(x, y) v(\xi, \eta) \mu_{1}(d x) \mu_{1}(d \xi) \mu_{2}(d y) \mu_{2}(d \eta)
$$

is absolutely convergent and its absolute value is not greater than 


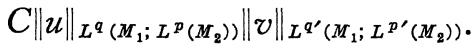

Proof. From Lemma 2.4 it follows that

$$
\iint|K(x, \xi, y, \eta) \| v(\xi, \eta)| \mu_{1}(d \xi) \mu_{2}(d \eta) \in L^{q^{\prime}}\left(M_{1} ; L^{p^{\prime}}\left(M_{2}\right)\right) .
$$

Therefore, making use of Hölder's inequality twice, we obtain the desired result.

In the following of this section $X$ will denote a Banach space and $B$ will denote the unit ball in $\boldsymbol{R}^{n}$.

Lemma 2. 6. Let $\Omega_{1} \subset \boldsymbol{R}^{n}, \Omega_{2} \subset \boldsymbol{R}^{m}, 1 \leqq q \leqq \infty-, 1 / q+1 / q^{\prime}=1$, $\lambda>0$, and let $K(x, y)$ be a measurable function satisfying.

$$
\begin{aligned}
& \sup _{x}\|K(x, y)\|_{L_{*}^{*} q^{\prime}\left(\Omega_{2}\right)}=C<\infty, \\
& \sup _{x}\|K(x, y)\|_{L_{*}{ }^{\prime}\left(\Omega_{2} \cap r|x| \lambda B\right)}=\varphi_{1}(r) \rightarrow 0 \text { as } r \rightarrow 0, \text { and } \\
& \sup _{x}\|K(x, y)\|_{L_{*} q^{\prime}\left(\Omega_{2} \backslash r|x| \lambda B\right)}=\varphi_{2}(r) \rightarrow 0 \text { as } r \rightarrow \infty .
\end{aligned}
$$

Then the integral operator $(T f)(x)=\int K(x, y) f(y) d_{*} y$ is a bounded operator from $L_{*}^{q}\left(\Omega_{2} ; X\right)$ into $L_{*}{ }^{\infty-}\left(\Omega_{1} ; X\right)$.

Proof. By Hölder's inequality we have

$$
\|T f\|_{L_{*}^{\infty}\left(\Omega_{1} ; X\right)} \leqq C\|f\|_{L_{*}^{q}\left(\Omega_{2} ; X\right)},
$$

and

$$
\begin{aligned}
\|T f(x)\|_{X} & \leqq\left\{\int_{\Omega_{2} \cap r|x| \lambda B}+\int_{\Omega_{2} \backslash r|x|^{\lambda} B}\right\}|K(x, y)|\|f(y)\|_{X} d_{*} y, \\
& \leqq \varphi_{1}(r)\|f\|_{L_{*}{ }^{q}\left(\Omega_{2} ; X\right)}+C\|f\|_{L_{*}{ }^{q}\left(\Omega_{2} \backslash r|x| \lambda B ; X\right)} .
\end{aligned}
$$

Since $q \leqq \infty$, it follows that $\|f\|_{L_{*}{ }^{q}\left(\Omega_{2}|r| x \mid{ }^{\alpha B}\right)} \rightarrow 0$ as $|x| \rightarrow \infty$, and therefore $\lim \sup _{|x| \rightarrow \infty}\|T f(x)\|_{X} \leqq \varphi_{1}(r)\|f\|_{L_{*}^{q}\left(\Omega_{2} ; X\right)}$. Letting $r \rightarrow 0$, we have $\|T f(x)\|_{X} \rightarrow 0$ as $|x| \rightarrow \infty$. Similarly we have $\|T f(x)\|_{X} \rightarrow 0$ as $|x| \rightarrow 0$, and the proof is complete.

Corollary 2. 7. Let $\Omega_{1} \subset \boldsymbol{R}^{n}, \quad \Omega_{2} \subset \boldsymbol{R}^{m}, \quad 1 \leqq p \leqq q \leqq \infty \quad$ (including $\infty-)$. And let 


$$
\begin{array}{ll}
\left(T_{1} f\right)(x)=\int_{|y| \geqq \rho|x|^{\lambda}}|x|^{\lambda \sigma}|y|^{-\sigma} f(y) d_{*} y & (\lambda, \rho, \sigma>0), \\
\left(T_{2} f\right)(x)=\int_{|y| \leqq \rho|x|^{\lambda}}|x|^{-\lambda \sigma}|y|^{\sigma} f(y) d_{*} y & (\lambda, \rho, \sigma>0)
\end{array}
$$

and

$$
\left(T_{3} f\right)(x)=\int \min \left\{|x|^{\sigma}|y|^{-\sigma},|x|^{-\tau}|y|^{\tau}\right\} f(y) d_{*} y(\sigma, \tau>0)
$$

for any $f \in L_{*}{ }^{p}\left(\Omega_{2} ; X\right)$. Then $T_{1}, T_{2}$ and $T_{3}$ are bounded operators from $L_{*}^{p}\left(\Omega_{2} ; X\right)$ into $L_{*}^{q}\left(\Omega_{1} ; X\right)$.

Proof. Let $\zeta_{\sigma}(t)=t^{\sigma}$ if $t \leqq 1$ and $\zeta_{\sigma}(t)=0$ if $t>1$. Then the kernel of $T_{1}$ is $\zeta_{\sigma}\left(\rho|x|^{\lambda} /|y|\right) \rho^{-\sigma}$ and that of $T_{2}$ is $\zeta_{\sigma}\left(|y| / \rho|x|^{\lambda}\right) \rho^{\sigma}$. It is easy to show that these kernels and $\min \left\{|x|^{\sigma}|y|^{-\sigma},|x|^{-\tau}|y|^{\tau}\right\}$ satisfy the conditions stated in Lemma 2.6 and [5] Lemma 2.5, which gives the desired result.

\section{§ 3. Besov Spaces $\boldsymbol{B}_{p, \infty-}^{\sigma}$}

In this section we discuss the properties of the Besov spaces $B_{p, \infty-}^{\sigma}$. Throughout this section $X$ is a Banach space.

Lemma 3. 1. If $1 \leqq p \leqq \infty, 1 \leqq q_{0} \leqq q_{1} \leqq \infty, j$ is a non-negative integer, $K(t, x, z, y) \in \mathcal{K}_{j}$, and if $\sigma<j$, then the mapping

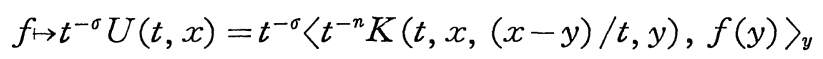

is a bounded linear operator from $B_{p, q_{0}}^{\sigma}(\Omega)$ into $L_{*}{ }^{q_{1}}\left([0, a] ; L^{p}(\Omega ; X)\right)$.

Proof. With the aid of Corollary 2.7, the same reasoning as in the proof of Lemma 4.1 in [6] gives the assertion.

Lemma 3. 2. If $1 \leqq p \leqq \infty, 1 \leqq q_{0} \leqq q_{1} \leqq \infty, j$ is a non-negative integer, $K(t, x, z, y) \in \mathcal{K}_{j}$, and if $-\sigma<j$, then the mapping

$$
u(t, x) \mapsto \int_{0}^{a} t^{\sigma} d_{*} t \int K(t, x,-z, x+t z) u(t, x+t z) d z
$$

is a bounded linear operator from $L_{*}^{q_{0}}\left([0, a] ; L^{p}(\Omega ; X)\right)$ into $B_{p, q_{1}}^{\sigma}(\Omega ; X)$. 
Proof. Corollary 2.7 and the same argument as in the proof of Lemma 4.2 in [6] give the conclusion.

By Lemma 3.1, Lemma 3.2 and the integral representation ([6] Theorem 1), we obtain the charaterization theorem for Besov spaces $B_{p, \infty-}^{\sigma}$. That is,

Theorem 3.3. Let $1 \leqq p \leqq \infty, \sigma \in \boldsymbol{R}$, and let $j$ be a non-negative integer with $j>\sigma$. Then $f \in B_{p, \infty_{-}}^{\sigma}(\Omega ; X)$ if and only if $f \in$ $W_{p}{ }^{-\infty}(\Omega ; X)$ and $t^{-\sigma}\left\langle t^{-n} K(x,(x-y) / t), f(y)\right\rangle_{y} \in L_{*}{ }^{\infty-}\left([0, a] ; L^{p}(\Omega ; X)\right)$ for any $K(x, z) \in \mathcal{K}_{j}$.

By Theorem 3.3 and Lemma 3.1 we have the following imbedding theorem (c.f. Remark in [6] p. 357).

Theorem 3.4. If $1 \leqq p \leqq \infty, 1 \leqq q \leqq \infty-$, then the imbedding operator

$$
B_{p, q}^{\sigma}(\Omega ; X) \rightarrow B_{p, \infty-}^{\sigma}(\Omega ; X)
$$

exists.

Let $1 \leqq q \leqq \infty-, u(t, x) \in L_{*}{ }^{q}\left([0, a] ; L^{p}(\Omega ; X)\right)$, and let for any $\varepsilon>0 u_{\varepsilon}(t, x)=u(t, x)$ if $\varepsilon \leqq t \leqq a$ and $u(t, x)=0$ if $t<\varepsilon$. Then $u_{\varepsilon}(t, x)$ $\rightarrow u(t, x)$ in $L_{*}{ }^{q}\left([0, a] ; L^{p}(\Omega ; X)\right)$ as $\varepsilon \rightarrow 0$. This fact, Lemma 3.1, Lemma 3.2 , Lemma 3.1 in [6], and Lemma 3.4 in [6], give the following theorem:

Theorem 3.5. (Approximation). Let $K(t, x, z, y) \in \mathcal{K}_{0}, \int K(0$, $x, z, x) d z=1, m$ be a positive integer,

$$
K_{m}(t, x, z, y)=\sum_{|\alpha|<m} \frac{1}{\alpha !} D_{z}^{\alpha}\left\{z^{\alpha} K(t, x, z, y)\right\},
$$

$1 \leqq p \leqq \infty, 1 \leqq q \leqq \infty-$, and let $\sigma<m$. Then for any $f \in B_{p, q}^{\sigma}(\Omega ; X)$

$$
U_{m}(t, x)=\left\langle t^{-n} K(t, x,(x-y) / t, y), f(y)\right\rangle_{y}
$$

converges to $f$ in $B_{p, q}^{\sigma}(\Omega ; X)$ as $t \rightarrow 0$, and for any $g \in B_{p, q, \bar{Q}}^{\sigma}\left(\boldsymbol{R}^{n} ; X\right)$

$$
V_{m}(t, x)=\left\langle t^{-n} K_{m}(t, y,(y-x) / t, x), g(y)\right\rangle_{y}
$$


converges to $g$ in $B_{p, q, \bar{a}}^{\sigma}\left(\boldsymbol{R}^{n} ; X\right)$ as $t \rightarrow 0$.

Since for $u \in L_{*}{ }^{\infty}\left([0, a] ; L^{p}\right) \quad u_{\varepsilon}$ does not converge to $u$ as $\varepsilon \rightarrow 0$ in $L_{*}{ }^{\infty}\left([0, a] ; L^{p}\right)$ unless $u \in L_{*}{ }^{\infty-}\left([0, a] ; L^{p}\right)$, the conclusion of Theorem 3.5 is not valid in case $q=\infty$. Consequently, the present author should have assumed that $q \leqq \infty-$ in [6] Theorem 5. Also, in the assertion that $C_{0}{ }^{\infty}$ is dense in $B_{p, q, \bar{\theta}}^{\sigma}\left(\boldsymbol{R}^{n}\right)$ ([6] p. 368) he should have assumed that $p<\infty$ and $q \leqq \infty-$

For a measurable set $M$ in $\boldsymbol{R}^{n}$ and a Banach space $X L_{*}^{p, \sigma}(M ; X)$ denotes the space of functions $f$ such that $|x|^{-\sigma} f(x) \in L_{*}^{p}(M ; X)$, and their norm is defined by

$$
\|f\|_{L_{x^{p}}^{p}, \sigma(M: X)}=\left\||x|^{-\sigma} f(x)\right\|_{L_{*}^{p}(M ; X)} .
$$

Let $X$ and $Y$ be Banach spaces contained in a Hausdorff vector space $E$. The mean interpolation space due to Lions and Peetre, which is denoted by $(X, Y)_{\theta, p}, 0<\theta<1,1 \leqq p \leqq \infty$, is the space of means

$$
f=\int_{0}^{\infty} u(t) d_{*} t, u \in L_{*}^{p,-\theta}\left(\boldsymbol{R}_{+} ; X\right) \cap L_{*}^{p, 1-\theta}\left(\boldsymbol{R}_{+} ; Y\right) .
$$

Lemma 3.6. Let $\sigma$ and $\tau$ be real numbers, $\sigma \neq \tau, 0<\theta<1, \mu$ $=(1-\theta) \sigma+\theta \tau$, and let $M$ be a measurable set in $\mathbb{R}^{n}$. Then

$$
\begin{aligned}
\left(L_{*}^{\infty, \sigma}(M ; X), L_{*}{ }^{\infty, \tau}(M ; X)\right)_{\theta, \infty-} & \subset L_{*}^{\infty-, \mu}(M ; X) \\
& \subset\left(L_{*}^{1, \sigma}(M ; X), L_{*}{ }^{1, \tau}(M ; X)\right)_{\theta, \infty-}
\end{aligned}
$$

with continuous injections.

Proof. Assume that $\lambda=\sigma-\tau>0$ (the case where $\lambda<0$ is discussed analogously). Let $f \in\left(L_{*}^{\infty, \sigma}(M ; X), L_{*}^{\infty, \tau}(M ; X)\right)_{\theta, \infty}$. Then there exists $u(t, x)$ such that

$$
f(x)=\int_{0}^{\infty} u(t, x) d_{*} t
$$

and $u(t, x) \in L_{*}^{\infty-,-\theta}\left(\boldsymbol{R}_{+} ; L_{*}{ }^{\infty, \sigma}(M ; X)\right) \cap L_{*}{ }^{\infty-1-\theta}\left(\mathbb{R}_{+} ; L_{*}{ }^{\infty, \tau}(M ; X)\right)$. It follows from this that

$$
\left\||x|^{-\mu} f(x)\right\|_{X} \leqq\left\{\int_{|x|^{2}}^{\infty}+\int_{0}^{|x|^{\alpha}}\right\}\left\||x|^{-\mu} u(t, x)\right\|_{X} d_{*} t,
$$




$$
\begin{aligned}
& \leqq \int_{|x|^{\lambda}}^{\infty}\left(|x|^{\lambda} / t\right)^{\theta}\left\|t^{\theta} u(t, x)\right\|_{L_{*}^{\infty}, \sigma(M ; X)} d_{*} t \\
& +\int_{0}^{|x|^{\lambda}}\left(|x|^{\lambda} / t\right)^{\theta-1}\left\|t^{\theta-1} u(t, x)\right\|_{L_{*}^{\infty}, \tau(M: X)} d_{*} t .
\end{aligned}
$$

Therefore, by Corollary 2.7 we have $\left\|x^{-\mu} f(x)\right\|_{X} \in L_{*}{ }^{\infty-}(M ; X)$. Conversely, let $f \in L_{*}{ }^{\infty-, \mu}(M ; X)$. Let $\varphi \in C_{0}\left(\boldsymbol{R}_{+}\right)$such that $\int_{\boldsymbol{R}_{+}} \varphi(t) d_{*} t=1$, and define $u(t, x)=\varphi\left(t|x|^{-\lambda}\right) f(x)$. Then it is obvious that

$$
f(x)=\int_{0}^{\infty} u(t, x) d_{*} t
$$

Since

$$
\left\|t^{\theta}|x|^{-\sigma} u(t, x)\right\|_{L_{*^{1}(M ; X)}}=\int \varphi\left(\left.t_{\mid}^{\prime} x\right|^{-\lambda}\right)\left(t|x|^{-\lambda}\right)^{\theta}\left\||x|^{-\mu} f(x)\right\|_{X} d_{*} x,
$$

and since the kernel $\varphi\left(t \mid x_{1}^{1-\lambda}\right)\left(t|x|^{-\lambda}\right)^{\theta}$ satisfies the conditions stated in Lemma 2.6 (this fact is a consequence of a simple calculation), it follows from Lemma 2.6 that $t^{\theta} u(t, x) \in L_{*}{ }^{\infty-}\left(\boldsymbol{R}_{+} ; L_{*}{ }^{1, \sigma}(M ; X)\right)$. The same observation gives that $t^{\theta-1} u(t, x) \in L_{*}{ }^{\infty-}\left(\boldsymbol{R}_{+} ; L_{*}{ }^{1, \tau}(M ; X)\right)$. Hence $f$ is an element of $\left(L_{*}{ }^{1, \sigma}(M ; X), L_{*}{ }^{1, \tau}(M ; X)\right)_{\theta, \infty-}$.

Theorem 3. 7. Let $\sigma$ and $\tau$ be real numbers, $\sigma \neq \tau, 0<\theta<1$, and let $\mu=(1-\theta) \sigma+\theta \tau$. Then

$$
\begin{aligned}
& \left(B_{p, q_{0}}^{\sigma}(\Omega ; X), B_{p, q_{1}}^{\tau}(\Omega ; X)\right)_{\theta, \infty-}=B_{p, \infty-}^{\mu}(\Omega ; X), \\
& \left(H_{p}{ }^{\sigma}(\Omega ; X), H_{p}^{\tau}(\Omega ; X)\right)_{\theta, \infty-}=B_{p, \infty-}^{\mu}(\Omega ; X)
\end{aligned}
$$

Proof. This follows from Lemma 3.6 and Theorem 3.3 (see Proof of [6] Theorem 8).

Theorem 3. 8. If $k$ is an integer, and if $1 \leqq p<\infty$, then $W_{p}{ }^{k}(\Omega)$ $\hookrightarrow B_{p, \infty-}^{k}(\Omega)$ with continuous injection.

Proof. Let $k \geqq 0, f \in W_{p}{ }^{k}(\Omega)$, and let $K(x, z)=\sum_{|\alpha|=k} D_{z}{ }^{\alpha} K(x, z)$, $K_{\alpha} \in \mathcal{K}_{1}$. Then, since $\int K_{\alpha}(x,-z) d z=0$,

$$
\left\langle t^{-k-n} K\left(x, \frac{x-y}{t}\right), f(y)\right\rangle_{y}=\sum_{|\alpha|=k} \int K_{\alpha}(x,-z)\left\{f^{(\alpha)}(x+t z)-f^{(\alpha)}(x)\right\} d z
$$


where $f^{(\alpha)}(x)=D^{\alpha} f(x)$. Since

$$
\left\|f^{(\alpha)}(x+y)-f^{(\alpha)}(x)\right\|_{L^{p} \rightarrow 0} \text { as }|y| \rightarrow 0,
$$

it follows that $\left\langle t^{-k-n} K(x,(x-y) / t), f(y)\right\rangle_{y} \in L_{*}^{\infty-}\left([0, a] ; L^{p}(\Omega)\right)$, and hence $f \in B_{p, \infty-}^{k}(\Omega)$. If $k<0, f \in W_{p}{ }^{k}(\Omega), K \in \mathcal{K}_{0}$, this facts follows from the identity

$$
\begin{aligned}
& \left\langle t^{-k-n} K\left(x, \frac{x-y}{t}\right), f(y)\right\rangle_{y} \\
& =\sum_{|\alpha| \leqq-k} \int t^{-|\alpha|-k} K^{(0, \alpha)}(x,-z)\left\{f_{\alpha}(x+t z)-f_{\alpha}(x)\right\} d z,
\end{aligned}
$$

where $f=\sum_{|\alpha| \leqq-k} D^{\alpha} f_{\alpha}, f_{\alpha} \in L^{p}(\Omega)$, and $K^{(0, \alpha)}(x, z)=D_{z}{ }^{\alpha} K(x, z)$.

\section{$\S 4$. Proof of the Main Results}

To prove the main results the following is fundamental.

Lemma 4. 1. Let $K(t, x, z, y) \in \mathcal{K}_{j}, H(t, x, z, y) \in \mathcal{K}_{i}$, and let

$$
J(s, t, x, y)=s^{-n} t^{-n} \int K\left(s, z, \frac{z-x}{s}, x\right) H\left(t, y, \frac{y-z}{t}, z\right) d z .
$$

Then,

$$
\sup _{y} \int|J(s, t, x, y)| d x, \sup _{x} \int|J(s, t, x, y)| d y \leqq C \min \left(s^{j} t^{-j}, t^{i} s^{-i}\right)
$$

holds for $0<s, t \leqq a<t_{0}$, where $C$ is a constant independent of $s, t$.

Proof. From the identity

$$
K\left(s, z, \frac{z-x}{s}, x\right)=s^{j} \sum_{|\beta| \leqq j}\left(-D_{z}\right)^{\beta}\left\{\widehat{K}_{\beta}\left(t, z, \frac{z-x}{t}, x\right)\right\}
$$

it follows that

$$
J(s, t, x, y)=\sum_{|\beta| \leqq j} s^{j-n} t^{-n} \int \widehat{K}_{\beta}\left(s, z, \frac{z-x}{s}, x\right) D_{z}{ }^{\beta}\left\{H\left(t, z, \frac{z-y}{t}, y\right)\right\} d z .
$$

This implies that

$$
\int|J(s, t, x, y)| d x \leqq C_{1} s^{j-n} t^{-j-n} \int_{y+t b B} d z \int_{z+s b B} d x=C s^{j} t^{-j}
$$


and

$$
\int|J(s, t, x, y)| d y \leqq C_{1} s^{j-n} t^{-j-n} \int_{x+s b B} d z \int_{z+t b B} d y=C s^{j} t^{-j}
$$

In the same way we have

$$
\int|J(s, t, x, y)| d y \leqq C t^{i} s^{-i}, \int|J(s, t, x, y)| d x \leqq C t^{i} s^{-i}
$$

Lemma 4. 2. Let $\sigma$ be a real number, $i$ and $j$ be non-negative integers, $-j<\sigma<i, 1 \leqq p \leqq \infty, 1 \leqq q \leqq \infty, 1 / p+1 / p^{\prime}=1,1 / q+1 / q^{\prime}=1$, $K_{k}{ }^{1}(t, x, z, y) \in \mathcal{K}_{j}, u_{k}(t, x) \in L_{*}{ }^{q}\left([0, a] ; L^{p}(\Omega)\right), k=1, \cdots, m_{1}, H_{h}{ }^{1}(t, x, z, y)$ $\in \mathcal{K}_{i}, v_{h}(t, x) \in L_{*}{ }^{q^{\prime}}\left([0, a] ; L^{p^{\prime}}(\Omega)\right), h=1, \cdots, l_{1}, K_{k}{ }^{0}(t, x, z, y) \in \mathcal{K}_{0}, f_{k}{ }^{0}(x)$ $\in L^{p}(\Omega), k=1, \cdots, m_{0}, H_{h}{ }^{0}(t, x, z, y) \in \mathcal{K}_{0}, \quad g_{h}{ }^{0}(x) \in L^{p^{\prime}}(\Omega), h=1, \cdots, l_{0}$, and let

$$
\begin{aligned}
f(x)= & \sum_{k} \int_{0}^{a} d_{*} t \int f^{-n+\sigma} K_{k}{ }^{1}\left(t, x, \frac{x-y}{t}, y\right) u_{k}(t, y) d y \\
& +\sum_{k} \int a^{-n} K_{k}{ }^{0}\left(a, x, \frac{x-y}{a}, y\right) f_{k}{ }^{0}(y) d y \\
g(x)= & \sum_{h} \int_{0}^{a} d_{*} t \int t^{-n-\sigma} H_{h}{ }^{1}\left(t, y, \frac{y-x}{t}, x\right) v_{h}(t, y) d y \\
& +\sum_{h} \int a^{-n} H_{h}{ }^{0}\left(a, y, \frac{y-x}{a}, x\right) g_{h}{ }^{0}(y) d y
\end{aligned}
$$

Then

$$
\begin{aligned}
& \sum_{k} \int_{0}^{a} d_{*} s \int s^{\sigma} u_{k}(s, x)\left\langle s^{-n} K_{k}{ }^{1}\left(s, y, \frac{y-x}{s}, x\right), g(y)\right\rangle_{y} d x \\
& \quad+\sum_{k} \int f_{k}{ }^{0}(x)\left\langle a^{-n} K_{k}{ }^{0}\left(a, y, \frac{y-x}{a}, x\right), g(y)\right\rangle_{y} d x \\
& =\sum_{h} \int_{0}^{a} d_{*} t \int t^{-\sigma} v_{h}(t, z)\left\langle t^{-n} H_{h}{ }^{1}\left(t, z, \frac{z-y}{t}, y\right), f(y)\right\rangle_{y} d z \\
& \quad+\sum_{h} \int g_{h}{ }^{0}(x)\left\langle a^{-n} H_{h}{ }^{0}\left(a, z, \frac{z-y}{a}, y\right), f(y)\right\rangle_{y} d z .
\end{aligned}
$$

Proof. By Lemma 2.2 Corollary 1 in [6] we have 


$$
\begin{aligned}
& \left\langle t^{-n} K_{k}{ }^{1}\left(s, y, \frac{y-x}{s}, x\right), g(y)\right\rangle_{y} \\
& =\sum_{h} \int_{0}^{a} d_{*} t \int s^{-n} K_{k}{ }^{1}\left(s, y, \frac{y-x}{s}, x\right) d y \int t^{-n-\sigma} H_{h}{ }^{1}\left(t, z, \frac{z-y}{t}, y\right) \\
& \quad \times v_{h}(t, z) d z \\
& \quad+\sum_{h} \int s^{-n} K_{k}{ }^{1}\left(s, y, \frac{y-x}{s}, x\right) d y \int a^{-n} H_{h}{ }^{0}\left(a, z, \frac{z-y}{a}, y\right) g_{h}{ }^{0}(z) d z \\
& =\sum_{h} \int_{0}^{a} d_{*} t \int J_{k h}^{11}(s, t, x, z) t^{-\sigma} v_{h}(t, z) d z+\sum_{h} \int J_{k h}^{10}(s, a, x, z) g_{h}{ }^{0}(z) d z
\end{aligned}
$$

where

$$
\begin{array}{r}
J_{k h}^{\nu \mu}(s, t, x, z)=s^{-n} t^{-n} \int K_{k}{ }^{\nu}\left(s, y, \frac{y-x}{s}, x\right) H_{h}{ }^{\mu}\left(t, z, \frac{z-y}{t}, y\right) d y, \\
(\nu, \mu=0,1)
\end{array}
$$

since for any $\varphi \in L^{p^{\prime}}(\Omega)$ the integrals

$$
\iint K_{k}{ }^{\nu}\left(s, y, \frac{y-x}{s}, x\right) H_{h}{ }^{\mu}\left(t, x, \frac{z-y}{t}, y\right) \varphi(x) d y d z, \quad(\nu, \mu=0,1)
$$

are absolutely integrable. Analogously, we can obtain

$$
\begin{aligned}
& \left\langle a^{-n} K_{k}^{0}\left(a, y, \frac{y-x}{a}, x\right), g(y)\right\rangle_{y} \\
& =\sum_{h} \int_{0}^{a} d_{*} t \int J_{k h}^{01}(a, t, x, z) t^{-\sigma} v_{h}(t, z) d z+\sum_{h} \int J_{k h}^{00}(a, a, x, z) g_{h}{ }^{0}(z) d z,
\end{aligned}
$$

and therefore the left hand side of $(5 \cdot 1)$ is equal to

$$
\begin{aligned}
\sum_{k, h} & \int_{0}^{a} d_{*} s \int u_{k}(s, x) d x \int_{0}^{a} d_{*} t \int s^{\sigma} t^{-\sigma} J_{k h}^{11}(s, t, x, z) v_{h}(t, z) d z \\
& +\sum_{k, h} \int_{0}^{a} d_{*} s \int u_{k}(s, x) d x \int s^{\sigma} J_{k h}^{10}(s, a, x, z) g_{h}^{0}(z) d z \\
& +\sum_{k, h} \int f_{k}^{0}(x) d x \int_{0}^{a} d_{*} t \int t^{-\sigma} J_{k h}^{01}(a, t, x, z) v_{h}(t, z) d z \\
& +\sum_{k, h} \int f_{k}^{0}(x) d x \int J_{k h}^{00}(a, a, x, z) g_{h}^{0}(z) d z .
\end{aligned}
$$

The same computation gives that its right hand side is equal to the sum 
of the same iterated integrals, except that the order of the integration is different. Thus, (4.1) follows from Fubini's theorem, if it is shown that the above integrals are absolutely integrable, which follows from Corollary 2.5 and Lemma 4.1 in view of the fact that

$$
\begin{aligned}
\int_{0}^{\infty} s^{\sigma} t^{-\sigma} \min \left(s^{j} t^{-j}, s^{-i} t^{i}\right) d_{*} t & =\int_{0}^{\infty} s^{\sigma} t^{-\sigma} \min \left(s^{j} t^{-j}, s^{-i} t^{i}\right) d_{*} s \\
& =\frac{1}{j+\sigma}+\frac{1}{i-\sigma} .
\end{aligned}
$$

Hence the proof of the lemma is complete.

Corollary 4.3. Let $\sigma, j, p, q, p^{\prime}, q^{\prime}, K_{k}{ }^{1}, u_{k}, K_{k}{ }^{0}, f_{k}{ }^{0}$ be as in Lemma 4.2, and assume that

$$
\begin{aligned}
\sum_{k} \int_{0}^{a} d_{*} t \int t^{\sigma-n} K_{k}{ }^{1}\left(t, x, \frac{x-y}{t}, y\right) u_{k}(t, y) d y \\
\quad+\sum_{k} \int a^{-n} K_{k}{ }^{0}\left(a, x, \frac{x-y}{a}, y\right) f_{k}{ }^{0}(y) d y=0
\end{aligned}
$$

Then

$$
\begin{aligned}
\sum_{k} \int_{0}^{a} d_{*} s \int s^{\sigma} u_{k}(s, x)\left\langle s^{-n} K_{k}{ }^{1}\left(s, y, \frac{y-x}{s}, x\right), g(y)\right\rangle_{y} d x \\
+\sum_{k} \int f_{k}{ }^{0}(x)\left\langle a^{-n} K_{k}^{0}\left(a, y, \frac{y-x}{a}, x\right), g(y)\right\rangle_{y} d x=0
\end{aligned}
$$

for every $g \in B_{p^{\prime}, q^{\prime}, \bar{\Omega}}^{-\sigma}\left(\mathbb{R}^{n}\right)$.

Proof. This follows from Lemma 4.2 and the integral representation ([6] Theorem 1).

Proof of Theorem 1.2. Let $\langle f, g\rangle$ be a continuous bilinear form with the property stated in the theorem and let $m, k, l, h$ be integers such that $m \geqq k \geqq 0, m-k>-\sigma, l \geqq h \geqq 0, l-h>\sigma$. Then, from the integral representation it follows that

$$
\begin{aligned}
f(x) & =\sum_{|\alpha|=k} \int_{0}^{a} d_{*} t \int t^{-n} M_{\alpha}\left(x, \frac{x-y}{t}\right)\left\{u^{\alpha}(t, y)+t^{k} f_{\infty}^{(\alpha)}(y)\right\} d y \\
& +\sum_{|\beta| \leqq h} \int_{0}^{a} d_{*} t \int t^{h-|\beta|-n} M^{(0, \beta)}\left(x, \frac{x-y}{t}\right) u_{\beta}(t, y) d y
\end{aligned}
$$




$$
\begin{aligned}
& +\sum_{|\alpha| \leqq h} \int a^{-|\beta|-n} \omega_{m}{ }^{(0, \beta)}\left(x, \frac{x-y}{t}\right) f_{\beta}(y) d y \\
& +\int a^{-n} \omega_{m}\left(x, \frac{x-y}{a}\right) f_{\infty}(y) d y
\end{aligned}
$$

(for the definition of $u^{\alpha}, u_{\beta}, f_{\beta}$ and $f_{\infty}$ see [6] p. 344) which gives

$$
\begin{aligned}
\langle f, g\rangle & =\sum_{|\alpha|=k} \int_{0}^{a} d_{*} t \int\left\{u^{\alpha}(t, x)+t^{k} f_{\infty}{ }^{(\alpha)}(x)\right\} V_{\alpha}(t, x) d x \\
& +\sum_{|\beta| \leqq h} \int_{0}^{a} d_{*} t \int t^{h-|\beta|} u_{\beta}(t, x) V^{\beta}(t, x) d x \\
& +\sum_{|\beta| \leqq h} \int f_{\beta}(x)(-1)^{|\beta|} g_{\infty}^{(\beta)}(x) d x+\int f_{\infty}(x) g_{\infty}(x) d x,
\end{aligned}
$$

where

$$
\begin{aligned}
& V_{\alpha}(t, x)=t^{-n}\left\langle M_{\alpha}(y,(y-x) / t), g(y)\right\rangle_{y}, \\
& V^{\beta}(t, x)=t^{-n}\left\langle M^{(0, \beta)}(y,(y-x) / t), g(y)\right\rangle_{y}, \\
& g_{\infty}(x)=a^{-n}\left\langle\omega_{m}(y,(y-x) / a), g(y)\right\rangle_{y}, g_{\infty}{ }^{(\alpha)}(x)=D^{\alpha} g_{\infty}(x) .
\end{aligned}
$$

Hence the bilinear form is unique. Conversely, the form defined by $(4 \cdot 2)$ is a continuous bilinear form on $B_{p, q}^{\sigma}(\Omega) \times B_{p^{\prime}, q^{\prime}, \bar{\Omega}}^{-\sigma}\left(\mathbb{R}^{n}\right)$ (see [6] Theorem 2 and [6] Lemma 3.3). Furthermore, it is obvious that the identities $(1 \cdot 1)$ and $(1 \cdot 2)$ follows from Corollary 4.3, and the identities $(1 \cdot 3)$ and (1.4) follows from Lemma 4.2. Thus Theorem 1.2 is completely proved.

Proof of Theorem 1.3. (i) Let $j>-\sigma$, and let $K \in \mathcal{K}_{j}$. Then for any $u(t, x) \in L_{*}^{q}\left([0, a] ; L^{p}(\Omega)\right)$

$$
\begin{aligned}
\int_{0}^{a} d_{*} t & \int t^{\sigma} u(t, x)\left\langle t^{-n} K\left(t, y, \frac{y-x}{t}, x\right), g(y)\right\rangle_{y} d x \\
= & \left\langle\int_{0}^{a} d_{*} t \int t^{\sigma-n} K\left(t, y, \frac{y-x}{t}, x\right) u(t, x) d x, g\right\rangle=0 .
\end{aligned}
$$

Hence, by Lemma 2.3 we have $\left\langle t^{-n} K(t, y,(y-x) / t, x), g(y)\right\rangle_{y}=0$. Also, if $K \in \mathcal{K}_{0}$ and if $f_{0} \in L^{p}(\Omega)$, then

$$
\int f_{0}(x)\left\langle a^{-n} K\left(a, y, \frac{y-x}{t}, x\right), g(y)\right\rangle_{y} d x
$$




$$
=\left\langle\int a^{-n} K\left(a, y, \frac{y-x}{t}, x\right) f_{0}(x) d x, g\right\rangle=0 \text {, }
$$

and therefore $\left\langle a^{-n} K(a, y,(y-x) / a, x), g(y)\right\rangle_{y}=0$. These facts imply that $g=0$, in view of the integral representation. The proof of part (ii) is analogous to that of (i).

Proof of Theorem 1. 4. Let $\langle f, g\rangle$ be the bilinear form on $B_{p, q}^{\sigma}(\Omega)$ $\times B_{p^{\prime}, q^{\prime}, \bar{\Omega}}^{-\sigma}\left(\boldsymbol{R}^{n}\right)$ defined in Theorem 1.2. It is already known that the mapping $g_{\mapsto} l_{g}$ and $f \mapsto l_{f}$ are continuous injections, where $l_{g}(f)=\langle f, g\rangle$ and $l_{f}(g)=\langle f, g\rangle$, so that it is sufficient to prove their surjectivity.

(i) For the case where $1<p<\infty, 1<q<\infty$ - this fact has been proved in [6].

(ii) Case $p=q=1$. Since the dual of $L^{1}\left([0, a] \times \Omega, d_{*} t d x\right)$ is $L^{\infty}\left([0, a] \times \Omega, d_{*} t d x\right)$, this fact can be proved in the same way as for the case (i).

(iii) Case $1<p<\infty, q=1$. Let $l \in\left\{B_{p, 1}(\Omega)\right\}^{\prime}$, and let $J$ be the imbedding operator from $B_{p, q}^{\tau}(\Omega)$ into $B_{p, 1}^{\sigma}(\Omega)$, where $\sigma<\tau$ and $1<q$ $<\infty-$ Then $l_{0} J \in\left\{B_{p, q}^{\tau}(\Omega)\right\}^{\prime}=B_{p^{\prime}, q^{\prime}, \bar{\Omega}}^{-\tau}\left(\boldsymbol{R}^{n}\right)$, so that there exists $g$ $\in B_{p^{\prime}, q^{\prime}, \bar{\Omega}}^{-\tau}\left(\boldsymbol{R}^{n}\right)$ such that $l_{0} J=l_{g}$. To prove $g \in B_{p^{\prime}, \infty, \bar{\Omega}}^{-\sigma}\left(\mathbb{R}^{n}\right)$, let $K \in \mathcal{K}_{j}$, $-\sigma<j, u(t, x) \in L_{*}{ }^{1}\left([0, a] ; L^{p}(\Omega)\right), \varepsilon>0$, and define

$$
u_{\varepsilon}(t, x)= \begin{cases}u(t, x) & \text { for } t \geqq \varepsilon, \\ 0 & \text { for } t<\varepsilon .\end{cases}
$$

Then $t^{\sigma-\tau} u_{\varepsilon}(t, x) \in L_{*}{ }^{q}\left([0, a] ; L^{p}\right)$, and therefore

$$
\begin{gathered}
\left\langle\int_{0}^{a} d_{*} t \int t^{\sigma-n} K\left(t, x, \frac{x-y}{t}, y\right) u_{\varepsilon}(t, y) d y, g\right\rangle \\
=\int_{0}^{a} d_{*} t \int t^{\sigma} u_{\varepsilon}(t, y) V(t, y) d y,
\end{gathered}
$$

where $V(t, y)=\left\langle t^{-n} K(t, x,(x-y) / t, y), g(x)\right\rangle_{x}$. Since

$$
|\langle f, g\rangle|=|l \circ J(f)| \leqq C\|J(f)\| B_{p, 1}^{\sigma},
$$

we have

$$
\left|\int_{\varepsilon}^{a} d_{*} t \int t^{\sigma} u(t, x) V(t, x) d x\right| \leqq C^{\prime}\|u\|_{L_{k^{1}}\left([0, a] ; L^{p}\right)} .
$$

Letting $\varepsilon \rightarrow 0$, by Lemma 2.3 we obtain that $t^{\sigma} V(t, x) \in L_{*}{ }^{\infty}\left([0, a] ; L^{p^{\prime}}(\Omega)\right)$. 
This and the fact that $g \in W_{p^{\prime}, \bar{\Omega}}^{-\infty}\left(\boldsymbol{R}^{n}\right)$ imply that $g \in B_{p^{\prime}, \infty, \bar{\Omega}}^{-\sigma}\left(\boldsymbol{R}^{n}\right)$, according to Theorem 2 in [6]. Therefore $l-l_{g}$ is continuous on $B_{p, 1}^{\sigma}(\Omega)$. Since $l-l_{g}=0$ on $J\left(B_{p, q}^{\tau}(\Omega)\right)$, which is dense in $B_{p, 1}^{\sigma}(\Omega)$ (see [6] Theorem 5), it follows that $l=l_{g}$ on $B_{p, 1}^{\sigma}(\Omega)$. Thus the mapping $g_{\mapsto} l_{g}$ is surjective.

(iv) Case $p=1,1<q<\infty-$. Making use of the imbedding operator $B_{1,1}^{\sigma}(\Omega) \rightarrow B_{1, q}^{\sigma}(\Omega)$, by the same argument as in case (iii) we obtain the desired result in this case.

(v) Case $1<p<\infty, q=\infty$ - With the aid of the imbedding $B_{p, r}^{\sigma}$ $\rightarrow B_{p, \infty-}^{\sigma}$, where $1<r<\infty-$, the same argument gives the desired conclusion.

(vi) Case where $p=\infty, 1 \leqq q \leqq \infty-$, and $\Omega$ is a bounded domain. Let $J$ be the imbedding $B_{r, q}^{\tau} \rightarrow B_{\infty, q}^{\sigma}$, where $1<r<\infty$ and $\tau=\sigma+n / r$, and let $l \in\left\{B_{\infty, q}^{\sigma}(\Omega)\right)^{\prime}$. Then $l_{\circ} J \in\left\{B_{r, q}^{\tau}(\Omega)\right\}^{\prime}=B_{r^{\prime}, q^{\prime}, \bar{\Omega}}^{-\tau}\left(\boldsymbol{R}^{n}\right)$, and hence there exists $g \in B_{r^{\prime}, q^{\prime}, \bar{\Omega}}^{-\tau}\left(\mathbb{R}^{n}\right)$ such that $l_{\circ} J=l_{g}$. Since $L^{r^{\prime}}(\Omega)$ is continuously imbedded in $L^{1}(\Omega)$, it follows that $g \in B_{1, q^{\prime}, \bar{\Omega}}^{-\tau}\left(\boldsymbol{R}^{n}\right) \subset W_{1, \bar{\Omega}}^{-\infty}\left(\boldsymbol{R}^{n}\right)$. The remainder part of the proof is the same as in case (iii).

Similarly we can find that the mapping $f \mapsto l_{f}$ is surjective, and the theorem is completely proved.

\section{References}

[1] Flett, T. M., Temperatures, Bessel potentials and Lipschitz spaces, Proc. London Math, Soc., (3) 20 (1970), 749-768.

[2] - Lipschitz spaces of functions on the circle and the disc, J. Math. Anal. Appl., 39 (1972), 125-158.

[3] Komatsu, H., Fractional powers of operators II, Interpolation spaces, Pacific J. Math., 21 (1967), 89-111.

[4] Muramatu, T., On Besov spaces of functions defined in general regions, Publ. RIMS, Kyoto Univ., 6 (1970), 515-543.

[5] - On imbedding theorems for Besov spaces of functions defined in general regions, Publ. RIMS, Kyoto Univ., 7 (1971), 261-285.

[6] - On Besov spaces and Sobolev spaces of generized functions defined on a general region, Publ. RIMS, Kyoto Univ., 9 (1974), 325-396.

[7] Zygumund, A., Smooth functions, Duke Math. J., 12 (1945), 47-76. 